\title{
Founder Effects Persist Despite Adaptive Differentiation: A Field Experiment with Lizards
}

Jason J. Kolbe, ${ }^{1 * \dagger}$ Manuel Leal, ${ }^{2}$ Thomas W. Schoener, ${ }^{3}$ David A. Spiller, ${ }^{3}$ Jonathan B. Losos ${ }^{1}$

${ }^{1}$ Department of Organismic and Evolutionary Biology and Museum of Comparative Zoology, Harvard University, 26 Oxford St., Cambridge, MA 02138, USA. ${ }^{2}$ Biology Department, Duke University, Durham, NC 27708, USA. ${ }^{3}$ Section of Evolution and Ecology and Center for Population Biology, One Shields Avenue, University of California Davis, Davis, CA 95616, USA.

*To whom correspondence should be addressed. E-mail: jjkolbe@gmail.com

†Present address: Department of Biological Sciences, University of Rhode Island, Kingston, RI 02881, USA.

The extent to which random processes such as founder events contribute to evolutionary divergence is a longstanding controversy in evolutionary biology. To determine the respective contribution of founder effects and natural selection, we conducted an experiment in which brown anole (Anolis sagrei) lizard populations were established on seven small islands from male-female pairs randomly drawn from the same large-island source. These founding events generated significant among-island genetic and morphological differences that persisted throughout the course of the experiment despite all populations adapting in the predicted direction-shorter hindlimbs - in response to the narrower vegetation on the small islands. Thus using a replicated experiment in nature we showed that both founder effects and natural selection jointly determine trait values in these populations.

Island populations are renowned for their extent of divergence from each other and from mainland source populations $(1,2)$. Mayr $(3,4)$ argued that these differences often are triggered by random sampling when island populations are founded by a few colonizing individuals. The resultant founder effects — changes in the genetic and phenotypic composition of a population due to founding by a small number of individuals - have been proposed as an important cause of evolutionary divergence and even speciation for the last half-century (3-6). However, an alternative explanation is that island environments differ from each other and the source locality, and that these ecological differences result in divergent natural selection (7-9). The evolutionary significance of founder effects also has been questioned because their imprint may be short-lived if populations perish due to lack of genetic variation or to demographic stochasticity, or if natural selection overwhelms their effects $(10,11)$. Data from nature are lacking because founder events are rarely observed [but see (12)], and thus their effects must be inferred post hoc; yet, laboratory studies indicate that even in the presence of natural selection, genetic drift induced by founder effects or population bottlenecks can contribute to patterns of phenotypic divergence [e.g., (13, 14)]. We report an experimental study of founder effects in a natural system of a Caribbean lizard, demonstrating that morphological divergence caused by the founder effect persists even as populations adapt to their new environments.

Some have argued that marked phenotypic change from a single founder event is very unlikely (11) and that genetic drift in general plays little part in morphological evolution (15). Moreover, others note that evidence from recent introductions suggests adaptation is a more common cause of trait evolution than drift (16-18). Yet, most concede that documenting these random sampling events in nature is exceedingly difficult $(11,15,19-21)$. Without knowledge of founder attributes and repeated sampling of colonized islands, the relative contributions of founder effects, population bottlenecks, natural selection, and gene flow to evolutionary divergence are impossible to disentangle (19-21).

We capitalized on the extensive knowledge of how Anolis lizards adapt to their environment, combined with the opportunity to use small islands, recently cleared of lizards by a hurricane, as experimental units in a natural setting. Our focus was on limb length, and the extent to which lizard populations would adapt to the novel environments on these small and scrubby islands. Extensive comparative and experimental research supports an adaptive explanation for the positive relationship between hindlimb length and perch diameter (i.e., the width of the cross section of the substrate that a lizard perches on, such as a branch or trunk) found among populations in anoles. Functional studies show a clear biomechanical basis: lizards with relatively longer hindlimbs run faster on broad substrates, whereas lizards with shorter limbs for a given body size move more adeptly on narrow surfaces. Such performance likely aids in capturing prey, defending territories, and escaping from predators. Indeed, field studies in this system show hindlimb length is under selection, favoring longer hindlimbs to run faster when exposed to terrestrial predators and shorter hindlimbs after becoming arboreal and shifting to narrower perches [reviewed in (22)]. 
To assess the relative importance of founder effects versus natural selection, we introduced lizards to replicate islands to which we predicted they were not well adapted due to differences in structural habitat from their source, thereby simulating founding events and altering the selective regime for limb length. Specifically, founding propagules were established on small experimental islands, all of which are sparsely vegetated, covered primarily with short, narrowdiameter vegetation compared to the more forested habitat of the source area on a nearby larger island (Fig. 1 and figs. S1 and S2). Our prediction is that if natural selection is the dominant force, then we would expect all populations to evolve shorter hindlimbs as they adapt to using narrower substrates; moreover, vegetation differences among experimental founder islands are expected to produce a relationship between hindlimb length and perch diameter [reviewed in (22)]. Conversely, if founder effects are dominant, then we would expect no general trend in limb length evolution, with some populations increasing in limb length and others decreasing with respect to the source population, and limb variation being unrelated to vegetation differences among islands.

In May 2005, we randomly selected one male and one female brown anole from lizards collected on a nearby larger island (Iron Cay, vegetated area $>150,000 \mathrm{~m}^{2}$ ) to found populations on seven small islands (vegetated area 35-175 $\mathrm{m}^{2}$ ). The storm surge from Hurricane Frances in September 2004 submerged these small islands near Great Abaco, Bahamas extirpating their lizard populations (23). We took morphological measurements and tissue samples for later genetic analysis from these founders. Over the next four years, we characterized the environment and repeatedly sampled lizards from the source population (Iron Cay), from the seven experimental founder islands, and from 12 nearby reference islands (vegetated area 74-324 $\mathrm{m}^{2}$ ) having A. sagrei and a composition of spider, insect, and plant species similar to that of the experimental islands (24). All lizard populations increased in size the first two years (averaging a 13-fold increase), and fluctuated in size thereafter (Fig. 2).

A founder effect was immediately apparent, conforming to theoretical expectations and previous empirical studies of genetic variation (27-29). In 2006, one year after introduction, multilocus genotypes from six microsatellite loci revealed an average decrease of $46 \%$ in allelic diversity and $23 \%$ in heterozygosity on experimental founder islands as compared to their source. Furthermore, allele frequencies differed significantly among all pairs of populations, including the source on Iron Cay (Stouffer's z method of combining probabilities from exact $G$ tests of six microsatellite loci; all $P<0.05$; table S1). Founder populations were dispersed around the mean value of the source population (Iron Cay) and varied five-fold in their amount of divergence from it; moreover, among-islands, genotype frequencies in subsequent years were correlated with the genotypes of the two founding individuals $(r=0.80$ 0.97 on principal coordinate (PCo) axes 1-3 for 2006, 2007, and 2009, all $P<0.05$; Fig. 3). Islands differed significantly from each other in genotype frequencies (ANOVAs for PCo1-3 separately, $F_{6,248}=16.5-41.7$, all $P<0.0001$ ), whereas both differences across years and among islands across years were not significant. In all but one case (the N3Iron Cay comparison), pairwise- $F_{\mathrm{ST}}$ values indicated significant genetic differentiation between islands (table S2), although $F_{\mathrm{ST}}$ values should be interpreted cautiously due to the likelihood of non-equilibrium conditions in these recently established populations. Moreover, genetic diversity was sustained after the founding event; only one locus out of 42 on the seven islands (six loci per island) showed a net decrease in the number of alleles from founding in 2005 to 2009. These results support persistence of the initial founder effect without subsequent genetic drift through time.

A founder effect also was evident for hindlimb length with experimental founder islands differing significantly in 2006 ( $P=0.0015$; table S3 and Fig. 4 inset). As with the genetic data, hindlimb values for island populations were dispersed around the mean value of the source population on Iron Cay (fig. S3). Furthermore, there was no relationship between perch diameter and relative hindlimb length among islands in $2006\left(r^{2}=0.15, P=0.39\right)$, supporting the interpretation that differences among islands in hindlimb length resulted from the founder event rather than adaptive divergence.

We used systematic transects to quantify the available structural habitat (i.e., vegetation height and diameter). The vegetation profile of the source population on Iron Cay averaged substantially taller and broader vegetation (mean vegetation height $=384 \mathrm{~cm}$ and mean vegetation diameter $=$ $11.1 \mathrm{~cm}$ ) compared to the experimental founder islands, which have much shorter and narrower vegetation (mean vegetation height ranged from $62-107 \mathrm{~cm}$ and mean vegetation diameter ranged from 1.6-5.4 cm) (Fig. 1 and figs. S1 and S2). In turn, the mean perch diameter used by lizards on all experimental founder islands $(3.2 \mathrm{~cm})$ was smaller than for the source population $(4.6 \mathrm{~cm})$.

As predicted by the adaptive relationship between hindlimb length and substrate diameter (22), relative hindlimb length decreased greatly over the course of three years on all seven experimental founder islands $(P<0.0001$; table S4 and Fig. 4 inset), with a decrease of $6.5 \%$ for males and $4.0 \%$ for females (calculated at the median body size for each sex; table S5 and fig. S4). The magnitude of this decrease in hindlimb length was so great that mean values in 2006 are nearly non-overlapping with 2009 values (Fig. 4 inset). We monitored nearby reference islands for the same length of time and the decrease on experimental founder islands was 
five times greater than on the reference islands (mixed effects ANCOVA test for an island type (reference vs. experimental founder islands) by year interaction: $P<0.0001$; table S6 and Fig. 4). The rates of decrease of all seven founders islands were greater than the rates for all reference islands, including the source population on Iron Cay (Fig. 4), and the mean rate for the reference islands did not differ significantly from zero ( $t=1.55$, d.f. $=12, P=0.15$ ), whereas the mean rate for the experimental founder islands did $(t=11.53$, d.f. $=6, P<$ 0.0001).

Despite this substantial adaptive response, the mark of the founder event persisted, as illustrated by the significant difference among islands (ANCOVA: $P<0.0001$; table S4) and minimal change in the order of mean hindlimb length values among experimental founder islands from 2006 to 2009 (year by island interaction: $P=0.70$; table S4 and fig. 4 inset). Furthermore, multiple regression confirms the persistence of the founder effect by showing that mean relative hindlimb lengths among islands in 2006 had a positive relationship with values in $2009(P=0.046)$, and demonstrates that no relationship existed between hindlimb lengths in 2009 and the diameter of either available vegetation ( $P=0.17$ ) or perch use $(P=0.86)$, indicating that adaptation to differing environments cannot explain variation among islands in hindlimb length.

Hindlimb growth can be affected by environment during ontogeny in A. sagrei (30), but such phenotypic plasticity is unlikely to explain the large decreases in hindlimb length detected on the experimental founder islands from 2006 to 2009. If plasticity was important, we would have expected to see an immediate difference in hindlimb length between the founders and their first-generation descendants due to the latter developing in the new, narrow-vegetation environment, but the range in population means in 2006 overlapped completely with the male founders. Moreover, perch diameter use did not change over time on experimental founder islands (year: $P=0.10$ and year by island interaction: $P=0.13$ ), even as hindlimb length progressively decreased, showing that the change in hindlimb length cannot be attributed to a change in a possible stimulus for plasticity. Finally, the magnitude of hindlimb divergence is 3-5 times greater than that produced by plasticity in $A$. sagrei raised on only narrow or broad surfaces in laboratory experiments (30) (fig. S5), even though the difference in perch diameter (1 versus $9 \mathrm{~cm}$ ) imposed in the laboratory experiment was much greater than the differences in mean perch-diameter use observed between the source population and experimental founder islands $(4.6 \mathrm{~cm}$ and 2.6-4.5 cm for Iron Cay and experimental founder islands, respectively).

Remarkably, founder events generated among-island genetic and phenotypic differences similar in magnitude to those observed among populations on nearby islands (our "reference” islands) as well as among natural populations occurring throughout the Bahamas (tables S1 to S3, Fig. 3, and figs. S3 and S5). The strong genetic differentiation among founder islands, illustrated by allelic differentiation and $\mathrm{F}_{\mathrm{ST}}$ values of similar magnitude to those seen among nearby reference islands, remained stable over the four years of the study (Mantel's $r=0.86-0.94$ for correlations among pairwise $\mathrm{F}_{\mathrm{ST}}$ matrices in 2006, 2007, and 2009, all $P<0.01$; tables S1 and S2). The strong imprint of the founder effect and stability of among-population genetic differentiation occurred despite evidence for ongoing immigration. Assignment tests and pedigree analyses revealed $11 \%$ of individuals sampled on experimental founder islands in 2007 and 2009 had multilocus genotypes consistent with being first-generation immigrants. In terms of phenotypic differentiation, the level of hindlimb divergence observed among experimental founder islands was only slightly less than that seen both among nearby reference islands and among natural populations from across the Bahamas (fig. S5). These results did not change when putative immigrants were removed from analyses. That the magnitude of the genetic and phenotypic differentiation produced by founder events rivals that seen among populations on natural islands indicates that founder effects may, as some have suggested, be an important cause of variation among islands (3-4).

Our results indicate that founder effects persist, even in the face of substantial adaptive differentiation. The impacts of founder events on neutral genetic variation have long been recognized and are clearly evident here $(27,29)$ (tables S1 and S2 and Fig. 3), but the significance of founder effects on phenotypic divergence is still debated. In support of the selectionist school, we show that differentiation from the source population is mostly the result of adaptation to the new environmental milieu on the experimental islands (7-9,1618,22). However, the imprint of the founder effect remains apparent, even as this adaptive divergence has occurred; indeed, variation among experimental founder islands at the present time is better explained by initial phenotypes than by current environmental conditions (Fig. 4 inset). What remains to be seen is whether-or for how long-these founder effects will persist in the face of natural selection. One might expect that just as selection has driven a decrease in hindlimb length from the source population in response to the narrower vegetation on the founder islands, it will ultimately also drive smaller-scale differentiation among islands as their lizard populations similarly adapt to the much smaller differences in perch diameter and erase the signal of the founder event. This prediction is not a foregone conclusion: not only are habitat differences among experimental founder islands small, but the ultimate outcome is dependent on the interaction of changing demographic, environmental, and genetic factors. 
We here show that founder effects can play an important role in island divergence, even as adaptation occurs. By measuring the phenotypic values of founders and their descendents, we have distinguished between influences of the founder effect and adaptation; thus we provide clear evidence of a rarely characterized dimension of evolutionary divergence among populations.

\section{References and Notes}

1. P. R. Grant, Ed, Evolution on Islands (Oxford Univ. Press, Oxford, 1998).

2. R. G. Gillespie, D. A. Clague, Eds, Encyclopedia of Islands (Univ. California Press, Berkeley, 2009).

3. E. Mayr, in Evolution as a Process, J. Huxley, A. C. Hardy, E. B. Ford, Eds. (Allen \& Unwin, London, 1954), pp. 157-180.

4. E. Mayr, Animal Species and Evolution (Harvard Univ. Press, Cambridge, 1963).

5. H. L. Carson, Stadler Genetics Symposium 3, 51 (1971).

6. A. R. Templeton, Genetics 94, 1011 (1980).

7. J. A. Endler, Natural Selection in the Wild (Princeton Univ. Press, Princeton, 1986).

8. N. H. Barton, in Evolution on Islands, P.R. Grant, Ed. (Oxford Univ. Press, Oxford, 1998), pp. 102-123.

9. D. Schluter, The Ecology of Adaptive Radiation (Oxford Univ. Press, Oxford, 2000).

10. T. Price, Speciation in Birds (Roberts \& Co. Publishers, Greenwood Village, 2007).

11. N. H. Barton, B. Charlesworth, Ann. Rev. Ecol. Syst. 15, 133 (1984).

12. P.R. Grant, B. R. Grant, K. Petren, Genetica 113, 359 (2001).

13. M. Travisano, J. A. Mongold, A. F. Bennett, R. E. Lenski, Science 267, 87 (1995).

14. P. Simões et al., Evolution 62, 1817 (2008).

15. J. A. Coyne, N. H. Barton, M. Turelli, Evolution 51, 643 (1997).

16. D. N. Reznick, C. K. Ghalambor, Genetica 112/113, 183 (2001).

17. S. M. Clegg et al., Evolution 56, 2090 (2002).

18. M. T. Kinnison, N. G. Hairston, Jr., Funct. Ecol. 21, 444 (2007).

19. C. W. Kilpatrick, in Mammalian Population Genetics, M. H. Smith, J. Joule, Eds. (Univ. Georgia Press, Athens, 1981), pp. 28-59.

20. R. J. Berry, in Evolution on Islands, P. R. Grant, Ed. (Oxford Univ. Press, Oxford, 1998), pp. 35-50.

21. P. R. Grant, Proc. Natl. Acad. Sci. U.S.A. 99, 7818 (2002).

22. J. B. Losos, Lizards in an Evolutionary Tree: Ecology and Adaptive Radiation of Anoles (Univ. California Press, Berkeley, 2009).
23. The patterns of lizard extinction were similar to those documented for Hurricane Floyd in T. W. Schoener, D. A. Spiller, J. B. Losos, Science 294, 1525 (2001).

24. D. A. Spiller, T. W. Schoener, Ecology 88, 37 (2007).

25. T. W. Schoener, D. A. Spiller, J. B. Losos, Ecol. Mono. 72, 383 (2002).

26. D. Reznick, H. Rodd, L. Nunney, in Evolutionary Conservation Biology, R. Ferrière, U. Dieckmann, D. Couvet, Eds. (Cambridge Univ. Press, Cambridge, 2004), pp. 101-118.

27. M. Nei, T. Maruyama, R. Chakraborty, Evolution 29, 1 (1975).

28. R. Chakraborty, M. Nei, Evolution 31, 347 (1976).

29. C. L. Tarr, S. Conant, R. C. Fleischer, Mol. Ecol. 7, 719 (1998).

30. J. B. Losos et al., Evolution 54, 301 (2000).

31. R. Calsbeek et al., Evol. Ecol. Research 9, 495 (2007).

32. T. W. Schoener, D. A. Spiller, J. B. Losos, Science 294,1525 (2001).

33. J. J. Kolbe et al., Biol. Lett. 4, 434 (2008).

34. R. Peakall, P. E. Smouse, Mol. Ecol. Notes 6, 288 (2006).

35. F. Rousset, GENEPOP (version 4.1) (2008).

36. M. C. Whitlock, J. Evol. Biol. 18, 1368 (2005).

37. D. Paetkau et al., Mol. Ecol. 13, 55 (2004).

38. S. Piry et al., J. Heredity 95, 536 (2004).

39. S. Manel et al., Trends Ecol. Evol. 16, 650 (2002).

40. E. Garcia-Berthou, J. Animal Ecol. 70, 708 (2001).

41. R. B. Darlington, T. V. Smulders, Animal Behav. 62, 599 (2001).

42. C. R. White, Physiol. Biochem. Zool. 76, 135 (2003).

43. P. Legendre, M. de Cáceres, D. Borcard, Ecology 91, 262 (2010).

44. J. B. Losos, D. J. Irschick, T. W. Schoener, Evolution 48, 1786 (1994).

45. J. B. Losos, K. I. Warheit, T. W. Schoener, Nature 387, 70 (1997).

Acknowledgments: We thank B. Pinder, J. Piovia-Scott, Y. Stuart, C. Thornber, and C. White for assistance; the National Geographic Society (8704-09), National Science Foundation (DEB-0444763 and DEB-0949415) and Duke University for funding; and the Bahamas Ministry of Agriculture and the Bahamas Environment, Science \& Technology (BEST) Commission of the Ministry of the Environment for permission to conduct this research.

\section{Supporting Online Material}

www.sciencemag.org/cgi/content/full/science.1209566/DC1 Materials and Methods

Figs. S1 to S5

Tables S1 to S6

References (31-45) 
9 June 2011; accepted 17 November 2011

Published online 2 February 2012; 10.1126/science.1209566

Fig 1. Vegetation differences between the source population and experimental founder islands. The schematic cartoon shows the change the vegetation profile for lizards introduced from the source population on the more forested Iron Cay to the sparsely vegetated experimental founder islands. The vegetation illustrated is scaled to the mean values of vegetation height for Iron Cay and the pooled experimental founder islands, respectively. Bar graphs show the difference (mean \pm SE) in available vegetation height and diameter from systematic transects on each island. We predicted the change in vegetation profile would result in lizards using narrower perches on experimental founder islands, which would alter the selective regime to favor shorter hindlimbs.

Fig. 2. Population size estimates for experimental founder islands. Surveys were conducted in May of each year from 2005-2009 [see (25) for details]. Population size showed a positive relationship with vegetated area of each island in $2006\left(r^{2}=0.53, P=0.06\right)$ and $2007\left(r^{2}=0.74, P=0.01\right)$, suggesting an effect of habitat area on population growth over the first two years. Population size estimates from November 2006, which included hatchlings produced that year, ranged from 21.4 to 60.2 (not shown in this figure). In all but one case, the November 2006 size estimates greatly exceeded the population size in May 2007, suggesting high mortality rates. Rapid population expansion such as found here may produce conditions favorable to evolution by natural selection $(16,26)$ and also curtail the loss of genetic variation after the initial founder effect.

Fig. 3. Multilocus genotypic variation for the source population and experimental founder islands. We conducted a principal coordinates (PCo) analysis of six microsatellite loci (mean \pm SE of PCo values). Numbers within each symbol correspond to different sampling years: I) 2005 (i.e., the founding pair on each experimental founder island and these same founder individuals pooled for the source population estimate on Iron Cay), II) 2006 (no Iron Cay estimate), III) 2007, and IV) 2009. Percentage of variation explained for each PCo axis is in parentheses. Stored sperm use was detected in $6 \%$ of offspring through 2006, resulting in offspring fathered by a male other than the one introduced onto the island. However, all other individuals sampled during this time had multilocus genotypes consistent with being progeny of the founding pair (or founding female in the case of stored sperm use) and not until 2007 do we detect unambiguous immigrants.

Fig. 4. Change in hindlimb length on reference and experimental founder islands. Change in mean relative hindlimb length (calculated as the residuals of the regression of log-hindlimb length on log-body length [i.e., snout-vent length], separately for each sex) per year for experimental founder and reference islands, including the source population on Iron Cay (open circle). The inset in the upper right shows how the mean $( \pm$ SE) relative hindlimb length decrease on the experimental founder islands in each year. Islands N1, N3, and X3* are shown with dotted lines for clarity. Immigration does not alter the results for hindlimb differentiation, which are virtually identical when putative immigrants are removed from analyses. Although the source population (Iron Cay) decreased in hindlimb length, all experimental founder islands exhibited even greater decreases than Iron Cay and all other reference islands with the mean rate of hindlimb change on reference islands not differing from zero. 


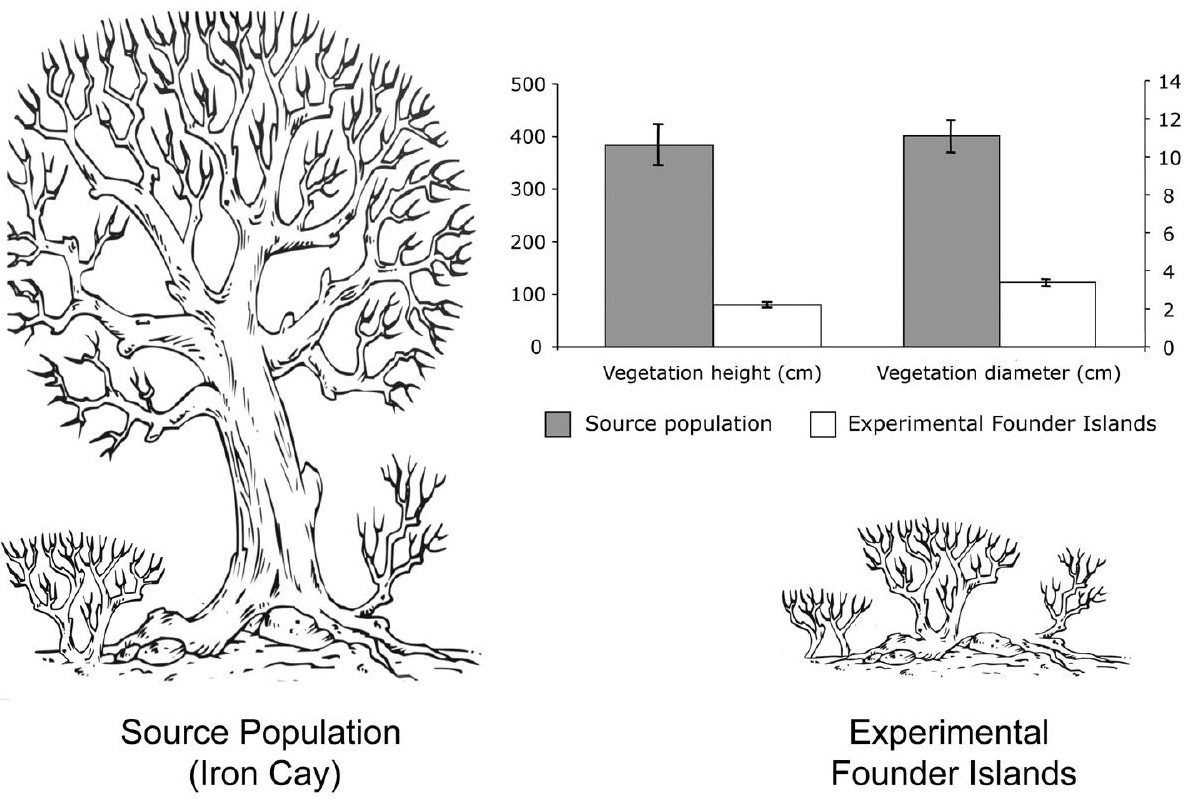




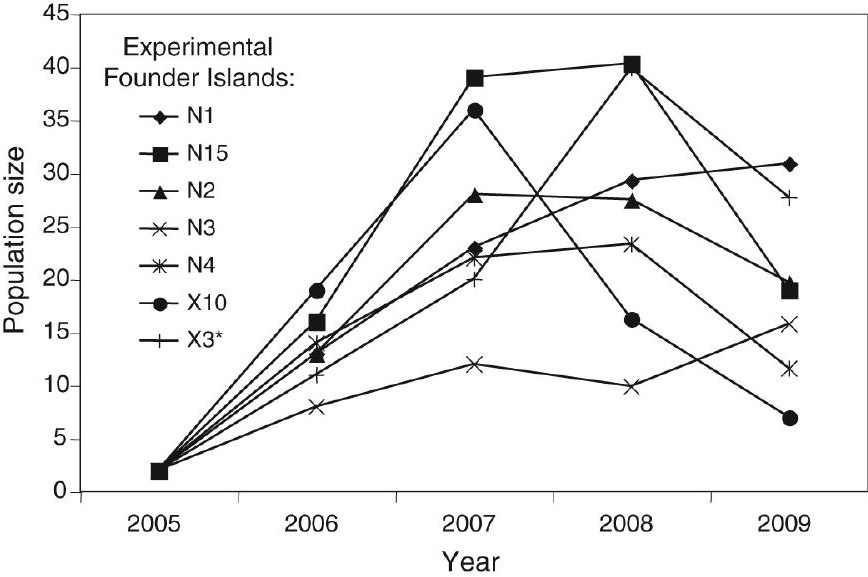




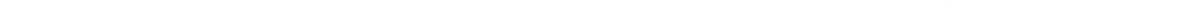

